ИЗВЕСТИЯ АКАДЕМИИ НАУК ЭСТОНСКОИ ССР. ТОМ 27 ФИЗИКА * МАТЕМАТИКА. 1978, № 1

\title{
УСЛОВИЯ КУНА-ТАККЕРА ДЛЯ ДВУХЭТАПНОЙ ЗАДАЧИ СТОХАСТИЧЕСКОГО ПРОГРАММИРОВАНИЯ С ИНТЕГРАЛЬНЫМИ ОГРАНИЧЕНИЯМИ
}

1. Рассмотрим задачу

$$
\begin{gathered}
F(x)=\int_{S} f_{10}\left(s_{1}, x_{1}\left(s_{1}\right)\right) \sigma\left(d s_{1}\right)+ \\
f \int_{S \times S} f_{20}\left(s_{1}, s_{2}, x_{1}\left(s_{1}\right), x_{2}\left(s_{1}, s_{2}\right)\right) \sigma\left(d s_{1}\right) \sigma\left(d s_{2}\right) \rightarrow \min ,
\end{gathered}
$$

$x_{1}\left(s_{1}\right) \in C_{1}$ почти всюду (п. в.),

$$
\begin{gathered}
\int_{S} f_{1 i}\left(s_{1}, x_{1}\left(s_{1}\right)\right) \sigma\left(d s_{1}\right) \leqslant 0, \quad i=1, \ldots, m_{1}, \\
x_{2}\left(s_{1}, s_{2}\right) \in C_{2} \text { п. в., } \int_{S \times S} \int_{2 i} f_{2 i}\left(s_{1}, s_{2}, x_{1}\left(s_{1}\right), x_{2}\left(s_{1}, s_{2}\right)\right) \sigma\left(d s_{1}\right) \sigma\left(d s_{2}\right) \leqslant 0, \\
i=1, \ldots, m_{2} .
\end{gathered}
$$

Обозначим через $(S, \Im, \sigma)$ основное вероятностное пространство. Пусть $C_{1} \subseteq R_{n_{1}}$ и $C_{2} \subseteq R_{n_{2}}-$ замкнутые с непустой внутренностью выпуклые множества. Предположим, что: 1) $f_{1 i}\left(s_{1}, \cdot\right), i=0, \ldots, m_{1}$, и $f_{2 i}\left(s_{1}, s_{2}, \cdot, \cdot\right), i=0, \ldots, m_{2}$, - конечные и выпуклые функции в пространствах $R_{n_{1}}$ и $R_{n_{1}} \times R_{n_{2}}$ соответственно для почти всех $s=$ $\left.=\left(s_{1}, s_{2}\right) \in S \times S ; \quad 2\right) \quad f_{1 i}\left(\cdot, x_{1}\right), i=0, \ldots, m_{1}$, и $f_{2 i}\left(\cdot, \cdot, x_{1}, x_{2}\right)$, $i=0, \ldots, m_{2}$, измеримые и суммируемые функции для всех $\left(x_{1}, x_{2}\right) \in R_{n_{1}} \times R_{n_{2}}$.

Решение $x=\left(x_{1}\left(s_{1}\right), x_{2}\left(s_{1}, s_{2}\right)\right)$ будем искать в пространстве $X=L_{n_{1}}^{p}(S) \times L_{n_{2}}^{p}(S \times S), \quad 1 \leqslant p \leqslant \infty$.

Целью данной статьи является выведение условий Куна-Таккера для характеризации решения задачи (1)-(3). По внешнему виду задача (1)-(3) напоминает обычную задачу программирования в функциональных пространствах, для которой теория необходимых условий хорошо разработана (см., напр., [ $\left.{ }^{1}\right]$ ). Отличает же стохастические задачи то обстоятельство, что решение их должно быть измеримым относительно выбранной $\sigma$-алгебры, которая описывает всю имеющуюся информацию. В модели (1)-(3) это выражается следующим образом.

На первом этапе решение $x_{1}$ является функцией от наблюдения $s_{1}$ при ограничениях (2). Потери измеряются функцией $f_{10}\left(s_{1}, x_{1}\left(s_{1}\right)\right)$. На 
втором этапе поступает следующее наблюдение $s_{2}$, и решение $x_{2}$ зависит уже от $s_{1}$ и $s_{2}$ при ограничениях (3). Так как ограничения (2) и (3) являются безусловными, т. е. ограничения (3) не зависят от наблюденного значения $s_{1}$ на втором этапе, то решение $x=\left(x_{1}\left(s_{1}\right)\right.$, $\left.x_{2}\left(s_{1}, s_{2}\right)\right)$ можно вычислить до наблюдений. Однако требование, что решение на первом этапе может зависеть только от $s_{1}$, представляет собой дополнительное ограничение в задаче (1)-(3), и это ограничение находит отражение в условиях Куна-Таккера.

Идея о том, что зависимость решения от разной информации есть дополнительное ограничение, которое вписывается в обычную схему теории минимизации, высказана в $\left[{ }^{2}\right]$. В $\left.{ }^{3-6}\right]$ была подробно исследована двухэтапная задача выпуклого стохастического программирования с зависящими от наблюдений ограничениями на втором этапе. В данной работе ограничения интегральные. При выводе соответствующих результатов мы воспользуемся методом и результатами из $[3,4]$.

Более общие результаты в этом направлении получены в $\left[{ }^{7,8}\right]$. Существование стохастического множителя Лагранжа в модели (1)-(3) вытекает из этих результатов.

2. Рассмотрим возмущенную задачу. Пусть $u \in U=R_{m_{1}} \times R_{m_{2}}$. Определим функцию $F: X \times U \rightarrow(-\infty, \infty]$ следующим образом. Если

$$
x_{1}\left(s_{1}\right) \in C_{1} \text { п. в., } \int_{\mathrm{S}} f_{1 i}\left(s_{1}, x_{1}\left(s_{1}\right)\right) \sigma\left(d s_{1}\right) \leqslant u_{1 i}, \quad i=1, \ldots, m_{1},
$$

и

TO

$$
\begin{gathered}
x_{2}\left(s_{1}, s_{2}\right) \in C_{2} \text { п. в., } \int_{S \times S} \int_{S} f_{2 i}\left(s_{1}, s_{2}, x_{1}\left(s_{1}\right), x_{2}\left(s_{1}, s_{2}\right)\right) \times \\
\times \sigma\left(d s_{1}\right) \sigma\left(d s_{2}\right) \leqslant u_{2 i}, \quad i=1, \ldots, m_{2},
\end{gathered}
$$

$F(x, u)=\int_{S} f_{10}\left(s_{1}, x_{1}\left(s_{1}\right)\right) \sigma\left(d s_{1}\right)+\int_{S \times S} \int_{S} f_{20}\left(s_{1}, s_{2}, x_{1}\left(s_{1}\right), x_{2}\left(s_{1}, s_{2}\right)\right) \sigma\left(d s_{1}\right) \sigma\left(d s_{2}\right)$.

В противном случае $F(x, u)=\infty$.

Рассмотрим задачу о минимизации функционала $F(x, u)$ по $x$. Задача $(1)-(3)$ заключается тогда в минимизации $F(x, 0)$.

Пусть $Y=L_{n_{1}}^{q}(S) \times L_{n_{2}}^{q}(S \times S), \frac{1}{p}+\frac{1}{q}=1 \quad$ и $V=R_{m_{1}} \times R_{m_{2}}$. Определим дуальность между $X \times U$ и $Y X V$ обычным соотношением

$$
\begin{gathered}
\langle(x, u),(y, v)\rangle=\int_{S}\left(x_{1}\left(s_{1}\right), y_{1}\left(s_{1}\right)\right) \sigma\left(d s_{1}\right)+ \\
+\int_{S \times S} \int_{S}\left(x_{2}\left(s_{1}, s_{2}\right), y_{2}\left(s_{1}, s_{2}\right)\right) \sigma\left(d s_{1}\right) \sigma\left(d s_{2}\right)+\sum u_{1 i} v_{1 i}+\sum u_{2 i} v_{2 i} .
\end{gathered}
$$

$$
A=\left\{x_{1}(\cdot) \in L_{n_{1}}^{p}(S): x_{1}\left(s_{1}\right) \in C_{1} \text { п. в. }\right\}
$$

является выпуклым и слабо (в случае $p=\infty-$ слабо ${ }^{*}$ ) замкнутым множеством в $L_{n_{1}}^{p}(S)$ (см. $\left[{ }^{9}\right]$, с. 357$)$.

Допустим, что

$$
\begin{array}{r}
\int_{\mathrm{S}} f_{1 i}\left(s_{1}, x_{1}\left(s_{1}\right)\right) \sigma\left(d s_{1}\right) \neq-\infty, \quad \forall x_{1}(\cdot) \in L_{n_{1}}^{p}(S), \\
i=0, \ldots, m_{1} .
\end{array}
$$

Тогда функционал $\int_{S} f_{1 i}\left(s_{1}, x_{1}\left(s_{1}\right)\right) \sigma\left(d s_{1}\right)$ - слабо полунепрерывен 
снизу в $L_{n_{1}}^{p}(S)$ (см. $\left.\left[{ }^{10}\right]\right)$ и поэтому его надграфик - слабо замкнутое множество относительно двойственности (4).

Если

$$
\begin{array}{r}
\int_{S \times S} f_{2 i}\left(s_{1}, s_{2}, x_{1}\left(s_{1}\right), x_{2}\left(s_{1}, s_{2}\right)\right) \sigma\left(d s_{1}\right) \sigma\left(d s_{2}\right) \neq-\infty, \\
\forall x \in X, i=0, \ldots, m_{2},
\end{array}
$$

то аналогичным свойством обладает множество, определяемое ограничением (3').

Л ем м а 1. Пусть выполнены условия (5) $u$ (5'). Тогда $F(x, u)$ является выпуклым и полунепрерывным снизу функционалом относительно двойственности (4).

Действительно, пусть ограничение (2') определяет множество $A_{1} \subset X \times U$. Функционал $F(x, u)$ можно записать в виде $F(x, u)=$ $=F_{1}(x, u)+F_{2}(x, u)$, где

$$
F_{1}(x, u)= \begin{cases}\int_{s} f_{10}\left(s_{1}, x_{1}\left(s_{1}\right)\right) \sigma\left(d s_{1}\right), & (x, u) \in A_{1} \\ \infty, & (x, u) \equiv A_{1} .\end{cases}
$$

Аналогично определяется $F_{2}(x, u)$.

Функционал $F_{1}(x, u)$ можно представить в виде

$$
F_{1}(x, u)=\int_{S} f_{10}\left(s_{1}, x_{1}\left(s_{1}\right)\right) \sigma\left(d s_{1}\right)+\chi_{A_{1}}, \text { где } \chi_{A_{1}}= \begin{cases}0, & (x, u) \in A_{1} ; \\ \infty, & (x, u) \equiv A_{1} .\end{cases}
$$

Первое слагаемое полунепрерывно снизу относительно двойственности (4). Так как $A_{1}$ замкнуто относительно (4), то $\chi_{A_{1}}$, а следовательно, и $F_{1}(x, u)$ тоже полунепрерывны снизу относительно (4).

Аналогично доказывается полунепрерывность снизу функционала $F_{2}(x, u)$.

Л ем м а 2. Пусть множества $C_{1}$ и $C_{2}$ ограничены. Тогда при каждом $\quad u \in U$ существует $\quad x \in L_{n_{1}}^{\infty}(S) \times L_{n_{2}}^{\infty}(S \times S)$, минимизирующее $F(x, u)$.

Лемма вытекает из леммы 1 и результатов [ $\left.{ }^{10}\right]$.

Определим функцию $\varphi(u)=\inf _{x \in X} F(x, u)=F(\bar{x}, u)$. Согласно лемме 1 , $\varphi(u)$ является выпуклой полунепрерывной снизу функцией.

Выпишем для задачи (1)-(3) функцию Лагранжа (ср. $\left.\left[{ }^{3}\right]\right)$.

$$
L(x, v)=\inf _{u \in U}\{\langle u, v\rangle+F(x, u)\}=
$$

$$
\begin{gathered}
=\sum v_{1 i} \int_{S} f_{1 i}\left(s_{1}, x_{1}\left(s_{1}\right)\right) \sigma\left(d s_{1}\right)+\sum v_{2 i} \int_{S \times S} f_{2 i}\left(s_{1}, s_{2}, x_{1}\left(s_{1}\right), x_{2}\left(s_{1}, s_{2}\right)\right) \times \\
\times \sigma\left(d s_{1}\right) \sigma\left(d s_{2}\right)+\int_{S} f_{10}\left(s_{1}, x_{1}\left(s_{1}\right)\right) \sigma\left(d s_{1}\right)+
\end{gathered}
$$

$+\int_{S \times S} \int_{S} f_{20}\left(s_{1}, s_{2}, x_{1}\left(s_{1}\right), x_{2}\left(s_{1}, s_{2}\right)\right) \sigma\left(d s_{1}\right) \sigma\left(d s_{2}\right), \quad$ если $x \in X_{0}, v \in V_{0}$; $=-\infty$, если $x \in X_{0}, v \equiv V_{0} ;=\infty$, если $x \equiv X_{0}$.

Здесь $X_{0}=\left\{x \in X: x_{1} \in C_{1}, x_{2} \in C_{2}\right.$ п. в. $\}, V_{0}=\left\{v \in V: v_{1} \geqslant 0, v_{2} \geqslant 0\right\}$.

Приспособим к рассматриваемой задаче (1)-(3) известные результаты общей теории двойственности (см., напр., $\left[{ }^{3,4}\right]$ ).

Функции Лагранжа соответствует следующая пара двойственных задач: 
$P:$ найти $x \in X$ такое, чтобы $\sup _{v \in V} L(x, v) \rightarrow \min$, и

$D:$ найти $v \in V$ такое, чтобы $\inf _{x \in X} L(x, v) \rightarrow \max$.

Задача $P$ эквивалентна задаче $(1)-(3)$.

Так как $\varphi(u)$ является полунепрерывной снизу функцией, то можно сказать, что $\min P=\sup D$. Если дополнительно предположить, что выполняется условие Слейтера (т. е. существует $x \in X$ такое, что

$$
x_{1}\left(s_{1}\right) \in C_{1} \text { п. в., } \int_{S} f_{1 i}\left(s_{1}, x_{1}\left(s_{1}\right)\right) \sigma\left(d s_{1}\right)<0, x_{2}\left(s_{1}, s_{2}\right) \in C_{2} \text { п. в., }
$$

$\left.\iint_{S \times S} f_{2 i}\left(s_{1}, s_{2}, x_{1}\left(s_{1}\right), x_{2}\left(s_{1}, s_{2}\right)\right) \sigma\left(d s_{1}\right) \sigma\left(d s_{2}\right)<0\right)$, то $\min P=\max D$.

Для решения задачи (1)-(3) получается следующая характеристика: $\bar{x} \in X$ является решением задачи (1)-(3) тогда и только тогда, когда существует $\bar{v} \in V$ такое, что $(\bar{x}, \bar{v})$ есть седловая точка функции Лагранжа.

3. Выведем условия Куна-Таккера, характеризующие седловую точку функции Лагранжа.

В силу леммы 2 решение $x=\left(x_{1}\left(s_{1}\right), x_{2}\left(s_{1}, s_{2}\right)\right)$ принадлежит пространству $L_{n_{1}}^{\infty}(S) \times L_{n_{2}}^{\infty}(S \times S)$. Предположим дополнительно, что для каждого $x_{1} \in L_{n_{1}}^{\infty}(S)$, удовлетворяющего условию (2), существует $x_{2} \in L_{n_{2}}^{\infty}(S \times S), \quad$ удовлетворяющий условию (3) (т. е. в задаче (1)-(3) нет индуцированных ограничений).

Пусть $(\bar{x}, \bar{v})$ - седловая точка функции Лагранжа, т. е.

$$
\max _{v \in V} L(\bar{x}, v)=L(\bar{x}, \bar{v})=\min _{x \in X} L(x, \bar{v}) .
$$

Т е о е м а. Для выполнения соотношения (6) необходимо и достаточно, чтобы:

a) $\bar{x}_{1}\left(s_{1}\right) \in C_{1}$ n. в., $\bar{v}_{1 i} \geqslant 0, \quad \int^{S} f_{1 i}\left(s_{1}, \bar{x}_{1}\left(s_{1}\right)\right) \sigma\left(d s_{1}\right) \leqslant 0$,

$$
\bar{v}_{1 i} \int_{S} f_{1 i}\left(s_{1}, \bar{x}_{1}\left(s_{1}\right)\right) \sigma\left(d s_{1}\right)=0, \quad i=1, \ldots, m_{1}
$$

б) $\bar{x}_{2}\left(s_{1}, s_{2}\right) \in C_{2}$ n.в., $\bar{v}_{2 i} \geqslant 0, \iint_{S \times S} f_{2 i}\left(s_{1}, s_{2}, \bar{x}_{1}\left(s_{1}\right), \bar{x}_{2}\left(s_{1}, s_{2}\right)\right) \sigma\left(d s_{1}\right) \sigma\left(d s_{2}\right) \leqslant$ $\leqslant 0, \quad \bar{v}_{2 i} \int_{S \times S} f_{2 i}\left(s_{1}, s_{2}, \bar{x}_{1}\left(s_{1}\right), \bar{x}_{2}\left(s_{1}, s_{2}\right)\right) \sigma\left(d s_{1}\right) \sigma\left(d s_{2}\right)=0, i=1, \ldots, m_{2} ;$ существует функция $\mathrm{Q}\left(s_{1}, S_{2}\right) \in L_{n_{1}}^{1}(S \times S)$ такая, что

в) функция $f_{10}\left(s_{1}, x_{1}\left(s_{1}\right)\right)+\sum v_{1 i} f_{1 i}\left(s_{1}, x_{1}\left(s_{1}\right)\right)+\left(x_{1}\left(s_{1}\right), \int_{S} \varrho\left(s_{1}, s_{2}\right) \sigma\left(d s_{2}\right)\right)$ достигает (при $x_{1}\left(s_{1}\right) \in C_{1}$ n.в.) своего минимума в точке $\bar{x}_{1}\left(s_{1}\right)$; г) функция $f_{20}\left(s_{1}, s_{2}, x_{1}\left(s_{1}\right), x_{2}\left(s_{1}, s_{2}\right)\right)+\sum v_{2 i} f_{2 i}\left(s_{1}, s_{2}, x_{1}\left(s_{1}\right), x_{2}\left(s_{1}, s_{2}\right)\right)$ - $\left(x_{1}\left(s_{1}\right), \quad \mathrm{e}\left(s_{1}, s_{2}\right)\right)$ достигает при $x_{1}\left(s_{1}\right) \in C_{1}, \quad x_{2}\left(s_{1}, s_{2}\right) \in C_{2}$ n. в. своего минимума в точке $\bar{x}=\left(\bar{x}_{1}\left(s_{1}\right), \bar{x}_{2}\left(s_{1}, s_{2}\right)\right)$.

Сформулированные условия (а) - (г) аналогичиы соответствующим условиям [ $\left.{ }^{4}\right]$. Условия (а) и (б) стандартные. Они обеспечивают выполнение первого равенства из (6). Достаточность (в) и (г) очевидна, 
покажем их необходимость. Для этого рассмотрим вычисление субдифференциала от одного интегрального функционала.

Пусть $X=L_{n_{1}}^{\infty}(S) \times L_{n_{2}}^{\infty}(S \times S), X_{1}=L_{n_{1}}^{\infty}(S \times S) \times L_{n_{2}}^{\infty}(S \times S)$

и пусть $f\left(s_{1}, s_{2}, x_{1}\left(s_{1}\right), x_{2}\left(s_{1}, s_{2}\right)\right)=f_{s}\left(x_{1}\left(s_{1}\right), x_{2}\left(s_{1}, s_{2}\right)\right)$ является выпуклым нормальным ннтегрантом (см. [11]) в пространстве $S \times S \times$ $\times R_{n_{1}} \times R_{n_{2}}$. Обозначим

$$
J f=\iint_{S \times S} f_{s}\left[x_{1}\left(s_{1}, s_{2}\right), x_{2}\left(s_{1}, s_{2}\right)\right] \sigma\left(d s_{1}\right) \sigma\left(d s_{2}\right),
$$

где $x=\left(x_{1}, x_{2}\right) \in X_{1}$.

Допустим, что $f\left(\cdot, \cdot, x_{1}, x_{2}\right)$ суммируема для всех $\left(x_{1}, x_{2}\right) \in$ $\in R_{n_{1}} \times R_{n_{2}}$. Известно [ [11], что тогда субдифференциал дJf функционала If принадлежит замкнутому подпространству сопряженного пространства $X_{1}^{\prime}$ - пространству суммируемых на $S \times S$ функций, а именно

$$
\partial J f=\partial f_{s}\left(x_{1}\left(s_{1}, s_{2}\right), x_{2}\left(s_{1}, s_{2}\right)\right) \subset Z=L_{n_{1}}^{1}(S \times S) \times L_{n_{2}}^{1}(S \times S) .
$$

Л ем м а 3. Пусть Јf является сужением функционала Jf на подпространство $X \subset X_{1}$. Тогда дЛf состоит из всех элементов вида $\tilde{z}=\left(\int_{S} z_{1}\left(s_{1}, s_{2}\right) \sigma\left(d s_{2}\right), z_{2}\left(s_{1}, s_{2}\right)\right)$, где $z=\left(z_{1}, z_{2}\right) \in \partial J f$.

Пусть $A: X \rightarrow X_{1}-$ канонический изоморфизм. Рассмотрим функционал $\mathcal{J f} \equiv \mathcal{J}^{A f}=\iint_{S \times S} f_{s}\left\{A\left[x_{1}\left(s_{1}\right), x_{2}\left(s_{1}, s_{2}\right)\right]\right\} \sigma\left(d s_{1}\right) \sigma\left(d s_{2}\right)$. Известно $\left({ }^{1}\right]$, с. 47), что $\partial \mathcal{J}^{A} f=A^{*} \partial J f$, где $A^{*}: X_{1}{ }_{1} \rightarrow X^{\prime}-$ сопряженный к $A$ оператор.

Пусть $X^{\perp}=\{z \in Z:(z, x)=0\}$ - аннулятор пространства $X$, т. е. $z \in X^{\perp} \subset Z, \quad$ если $\iint_{S \times S}\left(x_{1}\left(s_{1}\right), \quad z_{1}\left(s_{1}, s_{2}\right)\right) \sigma\left(d s_{1}\right) \sigma\left(d s_{2}\right)+\iint_{s \times S}\left(x_{2}\left(s_{1}, s_{2}\right)\right.$, $\left.z_{2}\left(s_{1}, s_{2}\right)\right) \sigma\left(d s_{1}\right) \sigma\left(d s_{2}\right)=0, \quad \forall x \in X . \quad$ Отсюда следует, что $X^{\perp}=$ $=\left\{z \in Z: \int_{S} z_{1}\left(s_{1}, s_{2}\right) \sigma\left(d s_{2}\right)=0, z_{2}\left(s_{1}, s_{2}\right)=0\right.$ п. в. $\}, X^{\prime}$ изометрично фактор-пространству $X_{1}^{\prime} / X^{\perp}\left(\left[{ }^{12}\right]\right.$, с. 269$)$. Но $X_{1}^{\prime} / X^{\perp}=\left\{\tilde{z}=\left(\tilde{z}_{1}, \tilde{z}_{2}\right) \in\right.$ $\left.\in Z \subset X_{1}^{\prime}: \tilde{z}_{1}=\int_{s} z_{1}\left(s_{1}, s_{2}\right) \sigma\left(d s_{2}\right), \quad \tilde{z}_{2}=z_{2}\left(s_{1}, s_{2}\right)\right\}, \quad$ что и доказывает лемму.

Доказательство необходимости условий (в) и (г) теоремы можно теперь провести по схеме работы [ $\left.{ }^{4}\right]$.

Если функции $f_{10}, f_{1 i}, f_{20}$ и $f_{2 i}$ дифференцируемы по $x=\left(x_{1}, x_{2}\right) \in$ $\in R_{n_{1}} \times R_{n_{2}}$ и $C_{1}=R_{n_{1}}, C_{2}=R_{n_{2}}$, то условия (в) и (г) принимают вид: существует функция $\mathrm{\varrho}\left(s_{1}, s_{2}\right) \in L_{n_{1}}^{1}(S \times S) \times L_{n_{2}}^{1}(S \times S)$ такая, что п. в.

$\left(\mathrm{B}^{\prime}\right) \nabla_{x_{1}} f_{10}\left(s_{1}, \bar{x}_{1}\left(s_{1}\right)\right)+\sum \bar{v}_{1 i} \nabla_{x_{1}} f_{1 i}\left(s_{1}, \bar{x}_{1}\left(s_{1}\right)\right)=-\int_{\mathrm{S}} \mathrm{Q}\left(s_{1}, s_{2}\right) \sigma\left(d s_{2}\right)$,

$\left(r^{\prime}\right) \nabla_{x_{1}} f_{20}\left(s_{1}, s_{2}, \bar{x}_{1}\left(s_{1}\right), \bar{x}_{2}\left(s_{1}, s_{2}\right)\right)+\sum \bar{v}_{2 i} \nabla_{x_{2}} f_{2 i}\left(s_{1}, s_{2}, \bar{x}_{1}\left(s_{1}\right), \bar{x}_{2}\left(s_{1}, s_{2}\right)\right)=$ $\nabla_{x_{2}} f_{20}\left(s_{1}, s_{2}, \bar{x}_{1}\left(s_{1}\right), \bar{x}_{2}\left(s_{1}, s_{2}\right)\right)+\sum \bar{v}_{2 i} \nabla_{x_{2}} f_{2 i}\left(s_{1}, s_{2}, \bar{x}_{1}\left(s_{1}\right), \bar{x}_{2}\left(s_{1}, s_{2}\right)\right)=0$.
$=0$.

Интерпретация функции $\varrho\left(s_{1}, s_{2}\right)$ дана в $\left[{ }^{4}\right] . \varrho\left(s_{1}, s_{2}\right)$ является множителем Лагранжа, что соответствует дополнительному ограничению $x_{1}\left(s_{1}\right)-\tilde{x}_{1}\left(s_{1}, s_{2}\right)=0$, т. е. функция $x_{1}\left(s_{1}\right)$ может зависеть только от $s_{1}$. В результате возмущения этого ограничения функцией из $L_{n}^{\infty}(S \times S)$ и появляется множитель Лагранжа $\varrho\left(s_{1}, s_{2}\right) \in$ $\in L_{n_{1}}^{1}(S \times S) \subset\left(L_{n_{1}}^{\infty}(S \times S)\right)^{\prime}$. 


\section{ЛИ ТЕ Р А Т У Р А}

1. Пшеничны й Б. Н., Необходимые условия экстремума, M., 1969.

2. Wets, R. J.-B., In: Intern. Symp. on Control Theory, Numerical Methods and Computer Systems Modelling, I. R. I. A., Springer-Verlag Lecture Notes, 107, 1974 , p. 350.

3. Rock a fella r, R. T., Wets, R. J.-B., Pacif. J. Math., 62, 173 (1976).

4. Rock a f e 11 a r R. T., Wets, R. J.-B., J. Math. Economics, 2, 349 (1975).

5. Rock a f ell a r R. T., W et s, R. J.-B., Pacif. J. Math., 62, 507 (1976).

6. R o ck a f e 11 a r, R. T., W e t s, R. J.-B., SIAM J. Control Opt., 14, 574 (1976).

7. Rock a f e 11 a r, R. T., Wets, R. J.-B., Math. Programming Study, 6, 170 (1976).

8. Evstigneev, I. V., Lecture Notes in Economics and Mathematical Systems, Springer-Verlag, 133, 1976, p. 34.

9. Иоффе А. Д., Тихомиров В. М., Теория экстремальных задач, М., 1974.

10. Пол як Б. Т., Матем. сборник, 78 (120), 65 (1969).

11. Rock a f e 11 a r, R. T., Pacif. J. Math., 39, 439 (1971).

12. Бу р баки Н., Топологические векторные пространства, М., 1959.

$\begin{array}{cc}\text { Институт кибернетики } & \text { Поступила в редакцию } \\ \text { Академии наук Эстонской ССР } & 20 / \text { XII } 1976\end{array}$

\section{KUHN-TUCKERI TINGIMUSED KAHEETAPILISELE INTEGRAALSETE KITSENDUSTEGA STOHHASTILISELE PROGRAMMEERIMISOLESANDELE}

On vaadeldud kaheetapilist stohhastilist programmeerimisülesannet kumera sihifuniktsiooni ja kumerate integraalsete kitsenduste korral ning tõestatud lahendi olemasolu teoreem. Kuhn-Tuckeri tingimuste tuletamisel on arvestatud lahendi järjestikust iseloomu, mistôttu tingimustesse on lisatud täiendav Lagrange’i kordaja.

\section{T. TOBIAS}

\section{KUHN-TUCKER CONDITIONS FOR TWO-STAGE STOCHASTIC PROGRAMMING MODEL WITH INTEGRAL CONSTRAINTS}

The two-stage stochastic programming model with convex objective and convex integral constraints is considered. The existence theorem is proved. On deriving the Kuhn-Tucker conditions, the sequential nature of the solution is taken into account and, as a result, the additional Lagrange multiplier appears. 